\title{
An alternative wind tunnel data correction based on CFD and experimental data in the transonic flow regime
}

\author{
Anna Bertram * and Stefan Görtz ${ }^{\dagger}$ \\ German Aerospace Center (DLR), Institute of Aeronautics and Flow Technologies, 38108 Braunschweig, Germany \\ Nils Hoffmann * \\ German Aerospace Center (DLR), Institute of Software Methods for Product Virtualization, 01069 Dresden, Germany \\ Roy Gebbink $\$$ \\ German-Dutch Wind Tunnels (DNW), Marknesse, 8316 PR, The Netherlands \\ Stevie Ray Janssen II \\ Netherlands Aerospace Centre (NLR), Amsterdam, 1059 CM, The Netherlands
}

\begin{abstract}
This paper presents an alternative approach to correct wind tunnel data through the use of CFD solutions. The correction is based on the difference between the measured pressures in the wind tunnel and the pressures predicted with CFD in free flow condition, at angles of attack and Mach numbers surround the wind tunnel data point. Optimization is used to find the combination of angle of attack and Mach number that minimizes the difference between the measured pressures and the predicted pressures. A surrogate model is used to approximate the CFD data to enhance the computational efficiency of the approach. The result of the optimization is a corrected angle of attack and Mach number that corresponds to a pressure distribution under free flight conditions that best fits the wind tunnel experiment in terms of the objective function used. It is demonstrated that when all wing pressures are used in the objective function the resulting corrections are in agreement with wall interference corrections as predicted with a wall pressure signature method.
\end{abstract}

\section{Nomenclature}

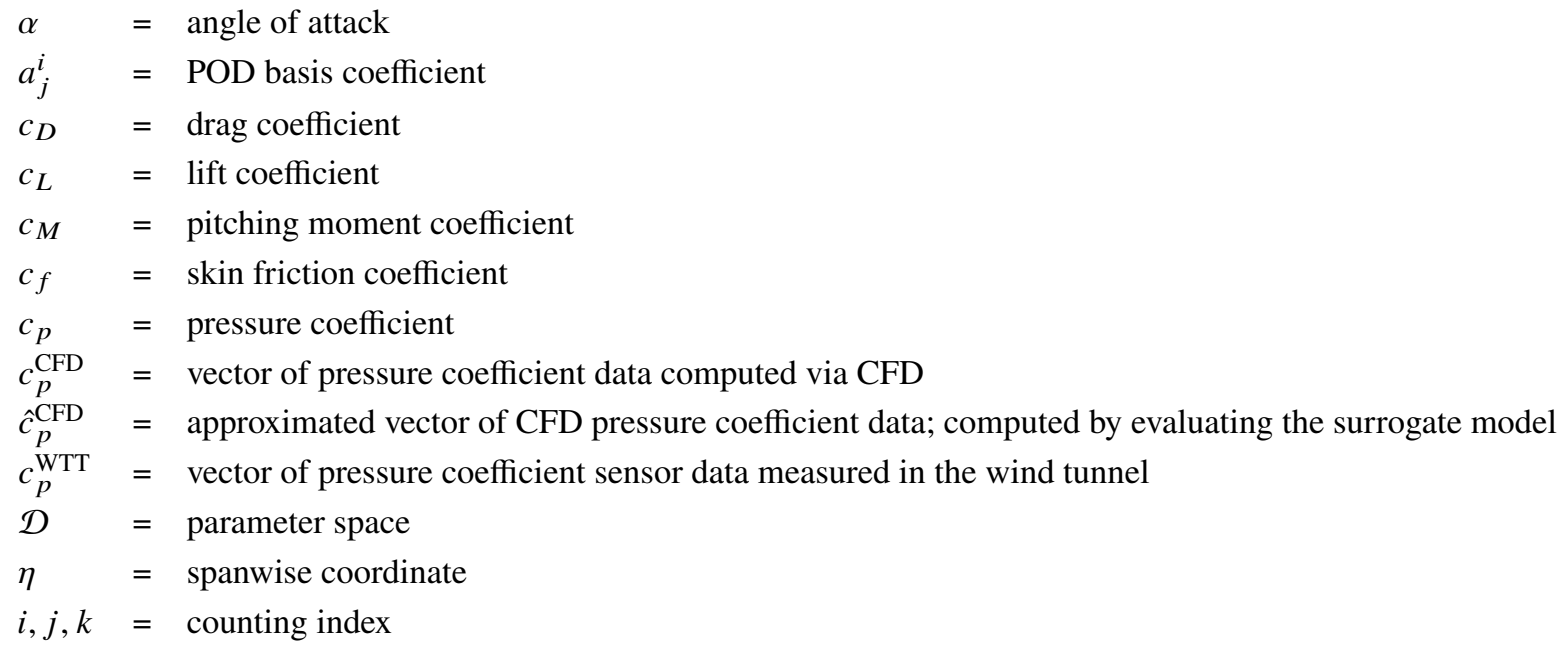

\footnotetext{
*Research Scientist, $\mathrm{C}^{2} \mathrm{~A}^{2} \mathrm{~S}^{2} \mathrm{E}$, anna.bertram@dlr.de

${ }^{\dagger}$ Head of department, $\mathrm{C}^{2} \mathrm{~A}^{2} \mathrm{~S}^{2} \mathrm{E}$, stefan.goertz@dlr.de

${ }^{\dagger}$ Student, $\mathrm{C}^{2} \mathrm{~A}^{2} \mathrm{~S}^{2} \mathrm{E}$, nils.hoffmann@ dlr.de

\$Project manager aerodynamics, roy.gebbink@dnw.aero

II Research Scientist, Flight Physics \& Loads, stevie.ray.janssen@nlr.nl
} 


$\begin{array}{ll}\mathcal{J} & =\text { index set } \\ \mathrm{M} & =\text { Mach number } \\ \operatorname{Re}_{c} & =\text { chord Reynolds number } \\ x, y, z & =\text { spatial coordinates } \\ y^{+} & =\text {dimensionless wall distance }\end{array}$

\section{Introduction}

Wind tunnel experiments are commonly used to investigate the aerodynamic behavior of flow bodies during the design stage. However, compared to an unbounded flow situation in the free atmosphere, wind tunnel measurements are influenced by experimental biases, like the presence of tunnel walls. For test sections with closed walls this leads to a compression of the streamlines, whereby the dynamic pressure and the velocity increase and larger forces and moments occur on the test object compared to the free flow situation. For test sections with ventilated walls blockage effects may be of opposite sign and are generally smaller compared to closed wall setups. However, in the transonic flow regime the advantage of somewhat smaller blockage effects is usually negated due to the increasing dependency of the sought-after aerodynamic coefficients on accurate Mach number setting. In order to compare results of wind tunnel tests with tests under free-flight conditions, corrections are therefore routinely applied.

Correcting for wall imposed biases is by no means trivial as the interference effects are dependent on aerodynamic characteristics of the test article itself. Extensive work [1] has gone into the assessment and formulation of generalized corrections via analytical solutions, semi-empirical methods and measured wall pressure signature methods [2, 3]. Drawbacks, however, are that the analytical and semi-empirical approaches are often inadequate for novel test objects of complex geometry. Wall pressure signature methods, on the other hand, are built from linearized potential flow theory which has shortcomings when compressibility and viscous effects become important.

Besides these more common approaches, there are also correction methods that make use of computational fluid dynamics (CFD) simulations. Examples are [4-8] where CFD codes are used to simulate a test model along with its support inside a virtual wind tunnel. Further CFD calculations are carried out without the support and tunnel walls to simulate the free flow situation at identical free stream conditions. The measured data is then corrected with the incremental differences from the different CFD solutions. Although these approaches have the potential to tackle all wall and/or support interference effects, they are computationally demanding and face a multitude of simulation challenges as summarized in [9, 10].

The so-called Gappy POD is a data fusion technique based on Proper Orthogonal Decomposition (POD), [11, 12], which aims to approximately reconstruct incompletely given data from a set of known data. It was originally proposed for the reconstruction of human face images in [13] and later further developed for fluid dynamic applications in [14]. Modified versions of the approach were introduced in [15-17] and successfully applied to reconstruct surface pressure coefficient distributions from wind tunnel sensor data and a set of pre-computed CFD simulations. The method was demonstrated to be able to accurately predict shock waves for industrial-relevant aircraft configurations in the transonic flow regime. However, as the aforementioned methods, it lacks of providing explicit correction values for the angle of attack and Mach number. An approach for the computation of correction values for these quantities is proposed in [18] in this case for the sake of validation and assessment of CFD codes. Correction values are determined by minimizing the relative error of the integral coefficients computed via CFD simulations with respect to the wind tunnel measured values. Following this procedure, good consistency regarding the overall integral coefficients could be achieved for a range of different turbulence models. However, for the assessment and correction of wind tunnel interference effects, one disadvantage of this procedure is that it only relies on the overall integral coefficients. Surface pressures, which are measured at the wind tunnel model, are not taken into account. Thus, the quality of predicting suction plateaus as well as the strength and position of occurring shock waves influence the target function only indirectly. A different approach, introduced in [19] and recently further developed in [20] and [21], seeks for the combination of Mach number and angle of attack for which the RANS CFD simulation result best matches the wind tunnel measured pressure coefficient data in a least-squares sense. To achieve this goal, adjoint methods are employed to compute the gradients of the associated loss function and iteratively correct the CFD flow solution towards the wind tunnel measurements. The approach was demonstrated to be able to accurately predict shock waves on airfoils in the transonic flow regime, [20, 21]. A drawback of this procedure, especially regarding more complex industrial applications, is that the direct use of the CFD solver in the optimization process is computationally highly demanding.

In this paper we address this issue by substituting the computationally expensive CFD solver by an interpolation-based 
surrogate model, which approximates target quantities in reasonable computational time. Considering CFD simulations for the model and its support under free flow conditions, we determine the combination of angle of attack and Mach number for which the CFD-based surface pressure distribution best matches the observed wind tunnel data. In order to enhance the efficiency of the optimization process, we replaced the CFD simulation by a surrogate model based on POD and interpolation in a data base of pre-computed CFD simulations, [22-26]. The potential of this approach for the investigation of wind tunnel interference effects is demonstrated exemplary for the wall interference on a transonic industrial-relevant test case where strong shocks are part of the flow solution. The resulting corrections are compared with wall interference corrections from a pressure signature method at a condition where it is known to produce reasonably reliable results.

After a description of the case study setup in Section III the correction approach is addressed in Section IV. Details on the surrogate modeling approach and the optimization task are given in Section IV.A and IV.B, respectively. Results of the case study are presented in Section V] followed by a discussion and conclusion in Section VI.

\section{Case study setup}

The case investigated in this study is the Aerodynamic Validation Model (AVM) by the Chinese Aeronautical Establishment (CAE). The CAE-AVM is a test-geometry of a conceptual intercontinental business jet, mainly designed for CFD validation. A scaled wind tunnel model was developed for the AVM, [27], and tested in the High-Speed Tunnel of DNW, Figure 1. The motivation for using the AVM in this study is the availability of $\mathrm{M}=0.85$ wind tunnel results, along with standardized CFD grids of the tested geometry. The standardized grids are available from the CAE-DNW workshop, [28], where it was demonstrated that by considering both wing deformation and support interference effects, the coincidence of CFD results with wind tunnel data improves significantly [29, 30]. The way this was demonstrated is via the inclusion of both the model support system and a deformed wing shape (as measured in the wind tunnel) in the CFD grid; thereby mimicking these experimental bias errors in the numerical simulation. The tunnel walls were not included in the CFD simulations. Instead their effects on the free stream conditions were corrected for the experimental data via an analysis of measured wall pressures [31].

In this work we take a different approach to determine how much the free stream conditions in the AVM test are influenced by the tunnel walls. The approach relies on CFD solutions, but it prevents the need for simulation of the full transonic test section. The idea is to use CFD predictions for the model with its sting support and a deformed wing shape (as measured in the wind tunnel) in free flow condition and determine the combination of angle of attack and Mach number for which the CFD predictions best match the wind tunnel data. To be more explicit: look for the $\alpha$-M combination for which the mean squared difference of wind tunnel data and CFD predictions at a specific sensor location (e.g. pressure distributions) is minimized. As the proposed approach essentially assumes CFD to be the unbiased truth, we purposefully limit the focus to conditions close to the AVM's design point: $\mathrm{M}=0.85, c_{L}=0.50$ and $\operatorname{Re}=4.7 \times 10^{6}$. The motivation being that the available computational grid is optimized for this condition and that any errors due to the turbulence model remain small as the flow is generally well-attached [9, 10]. In the end, the resulting $\Delta \mathrm{M}$ and $\Delta \alpha$ values are compared with the corrections from a wall pressure signature method.

\section{A. Wind tunnel data}

Experimental data for the CAE-AVM were obtained through testing in the High-Speed wind Tunnel of DNW (DNW-HST) in Amsterdam [32]. The DNW-HST is a closed-circuit variable pressure (0.25 bar to 4 bar) transonic wind tunnel with a Mach number range from 0.2 to 1.3 . The AVM was tested in the $1.8 \mathrm{~m} \times 2.0 \mathrm{~m}$ slotted test section, where the model was suspended via a ventral z-sting support as shown in Figure 1. Due to the size of the model, the wall interference effects should be modest: $68 \%$ of the test section width is taken up by the wing span and the frontal area occupies less than $1 \%$ of the test section cross sectional area.

The test comprised of measurements of the model overall forces and moments and wing pressure distributions. For an understanding to which geometry the measured forces, moments and pressures actually belong, the elastic deformation of the wing was measured as well. The wing pressure taps were located in six span-wise cuts parallel to the longitudinal axis of the fuselage, at $\eta=\{0.2,0.35,0.45,0.55,0.65,0.75\}$ as displayed in Figure 2.

The tests were conducted as sweeps of angle of attack at Mach numbers between 0.4 and 0.9 ; all at a constant Reynolds number of $\operatorname{Re}_{c}=4.7 \times 10^{6}$ based on the mean aerodynamic chord. On all relevant surfaces, laminar-turbulent transition tripping was forced using self-adhesive dots. Trip effectiveness was verified using infrared cameras as shown in [33].

For the present study, both uncorrected and wall interference corrected wind tunnel results are used. Specifically, the 


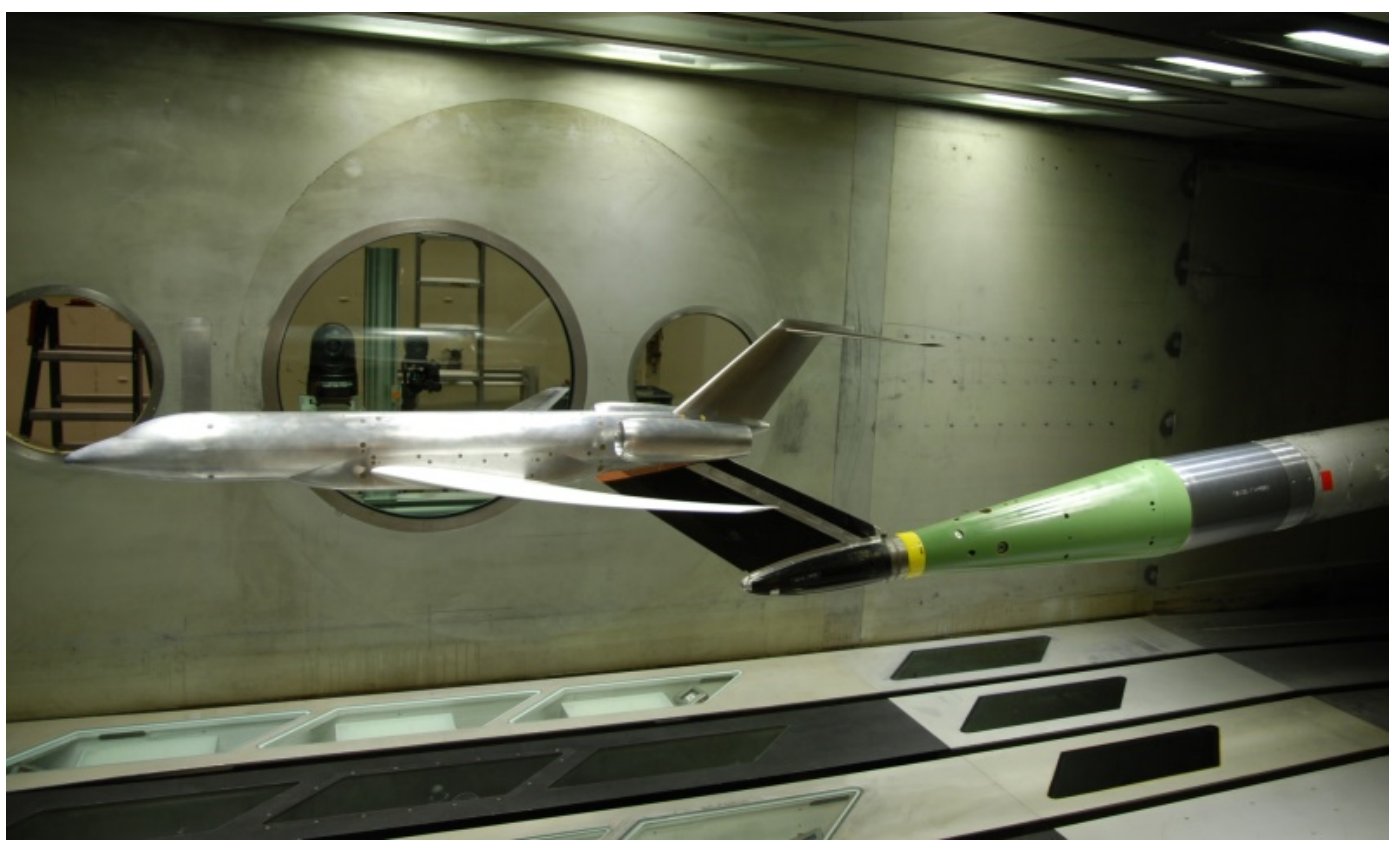

Figure 1 CAE-AVM installed in the DNW-HST.

overall load coefficients $c_{L}, c_{D}$ and $c_{M}$ as well as the pressure coefficients from 176 wing taps (distributed according to Table 1; ; at the flow condition $\mathrm{M}=0.850, \alpha=2.447^{\circ}$ and a Reynolds number of $4.7 \times 10^{6}$.

Table 1 Number and span-wise location of wing pressure taps.

\begin{tabular}{|c|c|c|}
\hline section cut & rel. span-wise location $\eta$ & number of taps \\
\hline 1 & 0.2 & 29 \\
2 & 0.35 & 29 \\
3 & 0.45 & 30 \\
4 & 0.55 & 30 \\
5 & 0.65 & 30 \\
6 & 0.75 & 29 \\
\hline
\end{tabular}

Details of the underlying instrumentation and their uncertainties are reported in [31]. The $\pm 2 \sigma$ uncertainty ranges for the aerodynamic coefficients and the free stream conditions are listed in Table 2. Note that these values reflect the uncertainty due to the instrumentation only; they do not account for the systematic errors due to aero elastic effects, support or wall interference. The bias errors due to wing deformation and support interference will be mimicked in the CFD simulations. They are therefore left undiscussed here, except for the remark that the so-called uncorrected data is acquired through the use of a standard blockage correction for the tunnel far-field, as defined in [34].

The CFD-based correction method proposed in this paper is compared to a one-variable measured wall pressure signature method which is usually used for the assessment and correction of wall interference effects. The method is called WIN3VE, [35], and it relies on measured wall pressure signatures, along with a model representation. The input wall signatures follow from wall pressure measurements with the installed model, tare corrected for the attributions of the test section and support system via the principle of superposition. The model representation is one that is built from potential flow singularities such as sources, sinks, line doublets and a vortex. The strengths of the singularities are sized via geometric properties of the model, along with the measured integral lift. The program combines these inputs to calculate the wall-induced disturbances in the vicinity of the model which are used to formulate corrections for angle of attack and Mach number, amongst others. As per normal convention [1] these corrections are defined as the disturbances in the wing $1 / 4$ and $3 / 4$-chord reference points, respectively. For the specific flow condition of this 


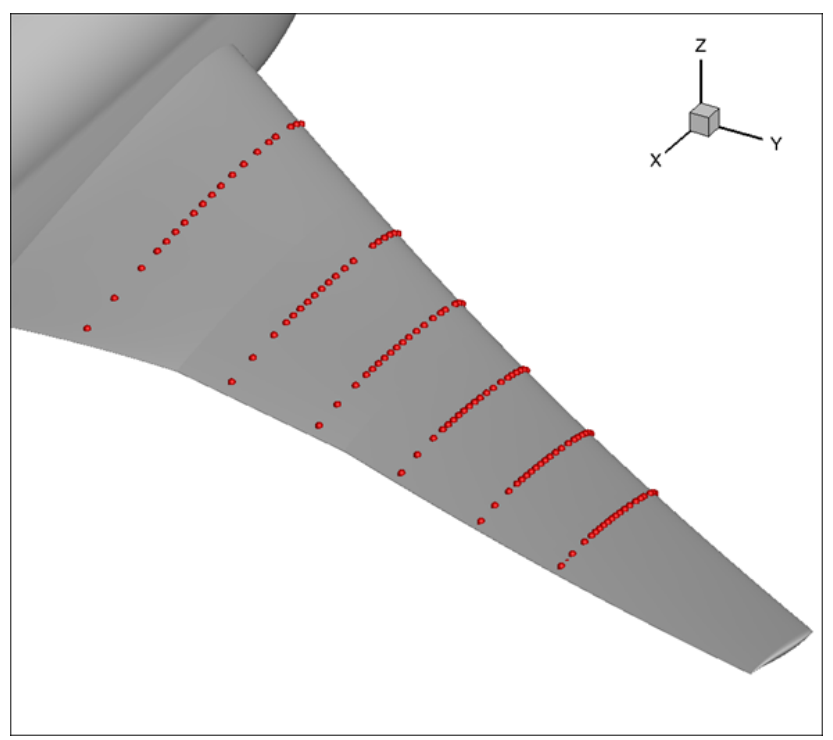

Figure 2 Position of the pressure taps on the upper surface of the wing.

Table $2 \pm 2 \sigma$ uncertainty ranges due to instrumentation.

\begin{tabular}{|c|c|}
\hline quantity & uncertainty \\
\hline$\alpha$ & $\pm 0.02^{\circ}$ \\
$\mathrm{M}$ & \pm 0.002 \\
$c_{L}$ & \pm 0.0038 \\
$c_{D}$ & \pm 0.0004 \\
$c_{M}$ & \pm 0.0013 \\
$c_{p}$ & \pm 0.0029 \\
\hline
\end{tabular}

study, WIN3VE yields estimates of $-0.04^{\circ}$ for the the wall-induced bias error on angle of attack and -0.001 for the free stream Mach number. The minuses here imply that the uncorrected values for $\mathrm{M}$ and $\alpha$ data were too high. Additional higher-order corrections to account for the fact that wall interference effects are not necessarily constant along the test article (e.g. along the span and/or fuselage) are not evaluated for this work.

\section{B. CFD setup}

The CFD simulations are performed on the AVM-DZ grid: a computational grid that was developed for the 2016 CAE-DNW workshop, [29, 36]. The AVM-DZ grid includes the aircraft model (wing, fuselage, pylons, through flow nacelles and tails) as well as the z-sting support used in the wind tunnel. The grid further contains a deformed wing shape, which corresponds to the measured spanwise twist and bending distributions in the wind tunnel at $\mathrm{M}=0.85$ and $\alpha=2.447^{\circ}$. Compared to the original wing, the added wing twist varies approximately linear from $0^{\circ}$ at root to $-1^{\circ}$ near the tip. The grid does not include the tunnel walls and the sting cavity inside the model is omitted. Geometric symmetry is assumed over the $y=0$ plane, which is why the grid employs the half-model, as shown in Figure 3.

The domain is discretized using a boundary conforming block-structured grid. The grid features wall stretching such that $y^{+}$is roughly equal to one in the first cell. It is designed such that at least 24 grid cells are contained in the boundary layer. The outer boundary, i.e. the artificial far-field boundary, is box-shaped with boundaries at $\pm 25 \mathrm{~m}$ from the origin, i.e. the intersection of the nose with the cylindrical part of the fuselage. The total grid consists of 9.9 million cells, distributed over 352 blocks for CPU load-balancing.

The flow solutions were obtained using NLR's in-house CFD code ENFLOW [37, 38], which solves the steady compressible RANS equations to simulate the flow in the given domain. The conservative form of the governing 


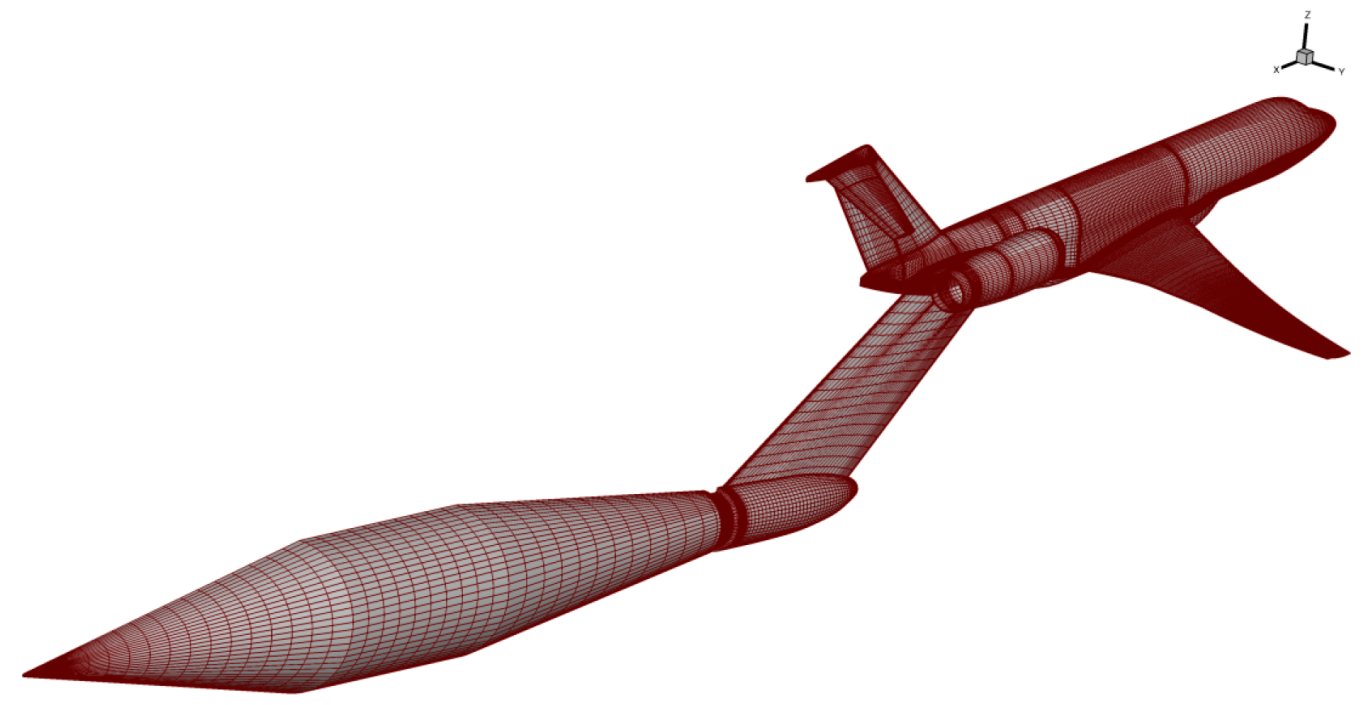

Figure 3 CFD surface mesh of the deformed half-aircraft, the AVM-DZ configuration.

equations is discretized using a cell-centred, second-order accurate, finite-volume method. Both 2nd and 4th order artificial diffusion is applied with a Jameson-type shock sensor for the basic flow equations and a TVD switch for the turbulence model equations. Turbulence is modelled using the explicit algebraic Reynolds-stress model (EARSM) with the characteristic turbulence length- and time scales determined using the $k-\omega$ model [39, 40]. Transition lines were specified on the various locations, i.e. nose, wing and tail leading edges, engine pylon, nacelle and sting. The specified lines correspond to the boundary layer tripping that was used in the experimental setup. The turbulence model is inactive in regions upstream of the specified transition lines.

The flow equations are solved via a multi-grid scheme, using a Runge-Kutta method as relaxation with local time stepping. Convergence was considered to be appropriate once variations of both force coefficients were below $10^{-4}$ on the finest grid level. This required up to 1500 iterations. A coarse grid solution was used as initial solution to accelerate the process.

CFD simulations were performed at small Mach number and angle of attack deviations around the baseline flow condition of $\mathrm{M}=0.85$ and $\alpha=2.447^{\circ}$. The Reynolds number and total temperature are set to identical values as in the experiment: $4.7 \times 10^{6}$ and $303 \mathrm{~K}$, respectively. A Quasi-Monte Carlo sampling process was used to randomly generate 15 parameter combinations within the two dimensional parameter space $(\mathrm{M}, \alpha) \in[0.84,0.86] \times\left[1.75^{\circ}, 3.00^{\circ}\right]$, shown in Figure 4

Note that the 15 flow cases are all computed on the same AVM-DZ grid which has a deformed wing shape that corresponds to a single flow condition. This is considered a fair approximation, given the expected magnitude of the eventual $\mathrm{M}$ and $\alpha$ corrections. 


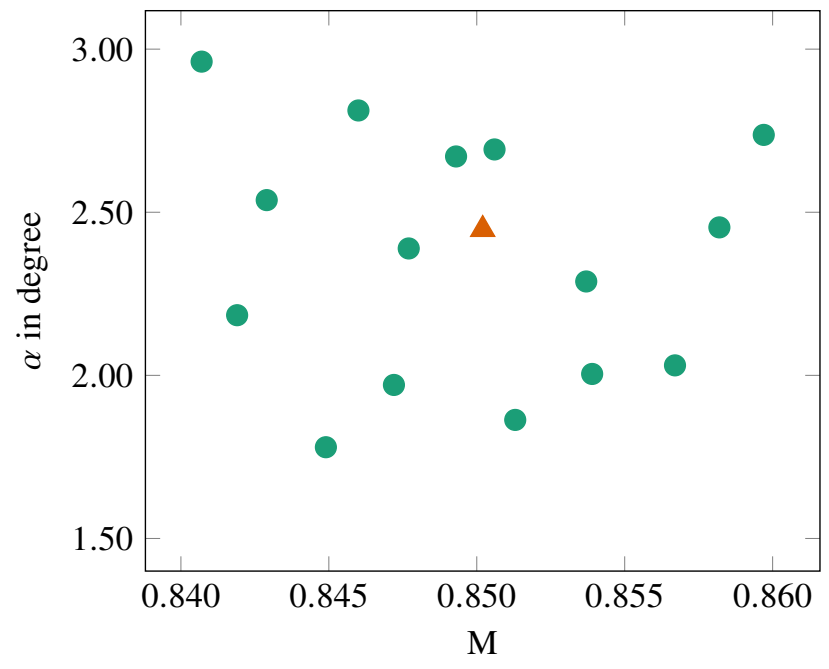

Figure 4 The 15 computed flow cases (green dots) and the wind tunnel data point (orange triangle).

\section{Mathematical description of the correction method}

To obtain the effective values for the angle of attack and Mach number, our approach is to compare the $c_{p}$-distribution as measured in the wind tunnel with CFD-based data in the least squares sense. The goal is to find the $\alpha$-M-combination for which the CFD simulation best matches the uncorrected wind tunnel data. Since high-fidelity CFD simulations are computationally expensive, their use in an optimization process is very demanding. To increase the efficiency of the process, direct CFD simulations are replaced by a data-driven surrogate model. A surrogate model is an inexpensive emulator of a given high-fidelity model, which approximates quantities of interest based on sampled data of the high-fidelity model. The surrogate modeling method is described in the next subsection, followed by a description of the correction procedure.

\section{A. The surrogate modeling approach}

The data-driven surrogate modeling approach chosen for the study at hand combines Proper Orthogonal Decomposition (POD) with interpolation techniques. It is commonly used in the context of aerodynamics in order to replace cost-intensive CFD simulations by an efficient emulator, see e.g. [22-25]. The approach is described in the following.

The CFD code is considered as a black-box model which maps the two input parameters, the angle of attack $\alpha$ and the Mach number $\mathrm{M}$, to the output vector representing the $c_{p}$ distribution,

$$
c_{p}^{\mathrm{CFD}}: \mathcal{D} \rightarrow \mathbb{R}^{N}, x=(\mathrm{M}, \alpha) \mapsto c_{p},
$$

where $\mathcal{D}=[0.84,0.86] \times[1.75,3.0]$ is the input space and $N=261,432$ is the number of grid points at the surface of the discretised aircraft model. Sample values of this mapping, so-called snapshots, are computed via CFD simulations,

$$
\mathcal{Y}=\left\{y^{1}, \ldots, y^{n}\right\}=\left\{c_{p}^{\mathrm{CFD}}\left(x^{1}\right), \ldots, c_{p}^{\mathrm{CFD}}\left(x^{n}\right)\right\},
$$

for different input parameters $x^{1}=\left(\mathrm{M}_{1}, \alpha_{1}\right), \ldots, x^{n}=\left(\mathrm{M}_{n}, \alpha_{n}\right)$. It is assumed that the span of the snapshots is a good approximation to the real output space of the black-box mapping, or with other words: The error of projecting a $c_{p}$-distribution, which is not included in the snapshot set, onto the span of the snapshots is assumed to be small. POD is used to compute an orthogonal basis $\left\{u^{1}, \ldots, u^{m}\right\} \subset \mathbb{R}^{N}$ of the snapshot space which is ordered by relative information content, that is the importance of the basis vectors to approximate the snapshots, [11]. If this basis is not truncated, every snapshot can be represented in the POD basis, i.e.

$$
\begin{aligned}
y^{1} & =c_{p}^{\mathrm{CFD}}\left(x^{1}\right)=a_{1}^{1} u^{1}+\ldots+a_{m}^{1} u^{m}, \\
& \vdots \\
y^{n} & =c_{p}^{\mathrm{CFD}}\left(x^{n}\right)=a_{1}^{n} u^{1}+\ldots+a_{m}^{n} u^{m},
\end{aligned}
$$


where the well-defined basis coefficients $a_{j}^{i}$ are given by the inner product

$$
a_{j}^{i}=\left\langle y^{i}, u^{j}\right\rangle, \quad i=1, \ldots, n, \quad j=1, \ldots, m .
$$

Since the basis coefficients are scalar-valued, classical interpolation techniques like polynomial interpolation, radial basis functions (RBF) or Kriging, can be used to obtain a model $\hat{a}_{j}(x)$ for every basis coefficient $a_{j}, j=1, \ldots, m$, based on the sampled data

$$
\left(x^{1}, a_{j}^{1}\right), \ldots,\left(x^{n}, a_{j}^{n}\right) .
$$

In this case study, these basis coefficient models were constructed using RBF interpolation with the thin plate spline (TPS) kernel $\phi(r)=r^{2} \ln (r)$, see [41] for further details. A prediction for the $c_{p}$-distribution at an untried input parameter combination $x^{*}=\left(\mathrm{M}_{*}, \alpha_{*}\right)$ is then given by the linear combination of the basis coefficient models evaluated at this point and the POD basis vectors,

$$
\hat{c}_{p}^{\mathrm{CFD}}\left(x^{*}\right)=\hat{a}_{1}\left(x^{*}\right) u^{1}+\cdots+\hat{a}_{m}\left(x^{*}\right) u^{m} .
$$

The overall workflow is shown schematically in Figure 5
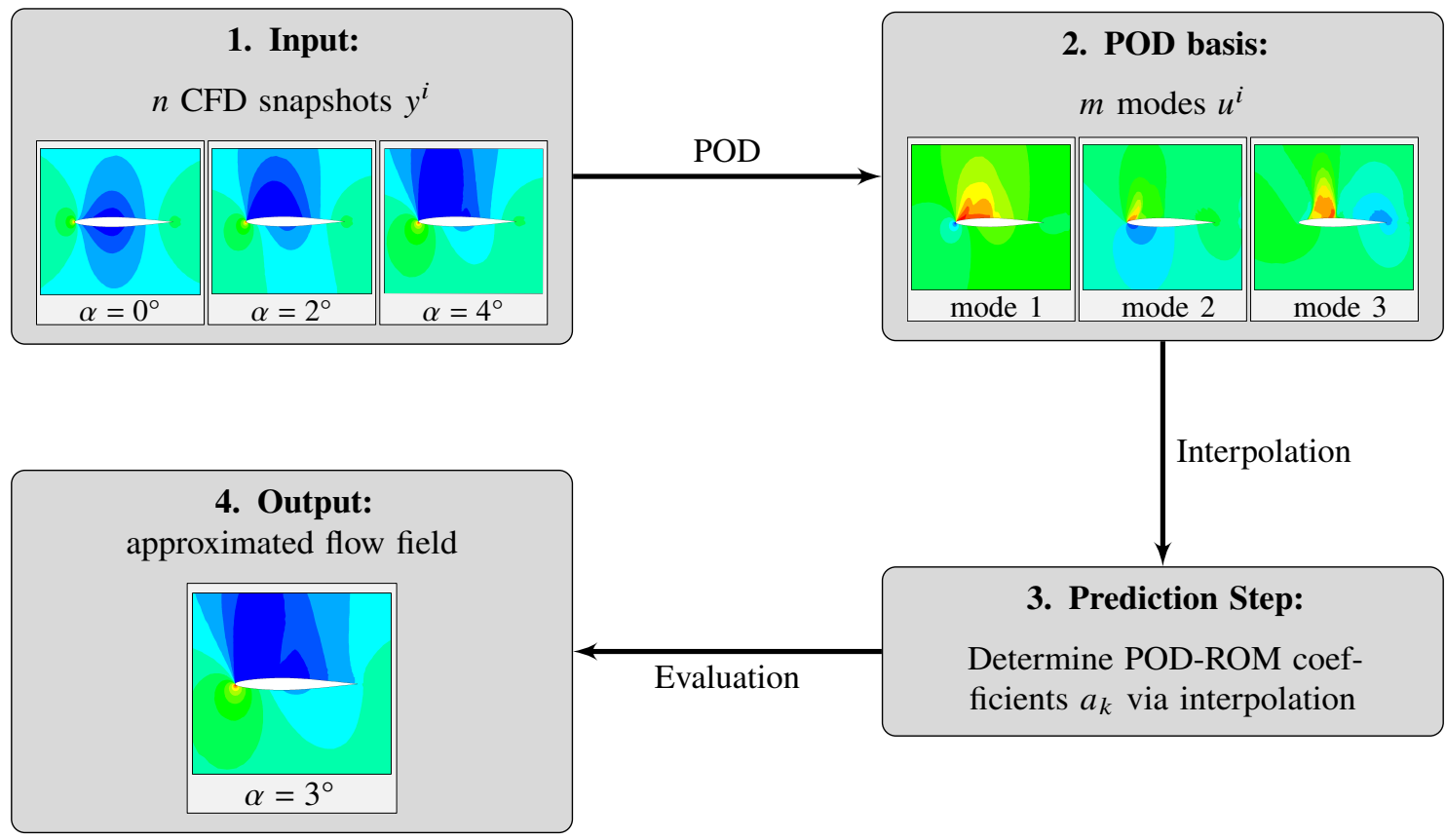

Interpolation

Figure 5 Schematic representation of the surrogate modeling process.

Since the evaluation of all coefficient models requires the solution of a linear system with fixed system matrix of dimension $n \times n$ for multiple right hand sides, [41, p. 164], the complexity of evaluating the models is $O\left(n^{3}\right)$. In the case study at hand, where $n=15$ sample CFD solutions are given, the evaluation of the coefficient models and thus the computation of the linear combination of the POD basis vectors can be done on usual modern desktop computers in real-time.

\section{B. The correction procedure}

While the CFD solutions are defined at the nodes of the computational grid, the pressure taps can have arbitrary positions on surface cells between several grid points. Therefore, an interpolation scheme is needed to compare the CFD data and the wind tunnel measurements at the pressure taps. Since the computational grid in the case at hand features a fine resolution, the simple nearest neighbor interpolation method, i.e. constant interpolation, yields sufficiently good approximations. For every pressure tab location, the nearest neighbor method returns the CFD solution at the closest node of the computational grid. 
Let $I(i) \in\{1, \ldots, N\}$ denote the index of the node, which is closest to the $i$-th pressure tab and let $\left(c_{p}^{\mathrm{CFD}}(x)\right)_{I(i)}$ be the corresponding entry of the CFD solution vector $c_{p}^{\mathrm{CFD}}(x)$ for input parameter $x=(\mathrm{M}, \alpha)$. The error between CFD result and wind tunnel measurement $\left(c_{p}^{\mathrm{WTT}}\right)_{i}$ at pressure tab $i$ is given by

$$
e_{i}(x):=\left|\left(c_{p}^{\mathrm{WTT}}\right)_{i}-\left(c_{p}^{\mathrm{CFD}}(x)\right)_{I(i)}\right| .
$$

To quantify the difference between the CFD results and the wind tunnel measurements at a set of pressure taps $\mathcal{J}$, the root mean squared error (RMSE),

$$
\operatorname{RMSE}_{\mathcal{J}}(x):=\sqrt{\frac{1}{|\mathcal{J}|} \sum_{i \in \mathcal{J}}\left(e_{i}(x)\right)^{2}}
$$

is considered. Since the direct use of CFD in a numerical optimization of the RMSE is computationally very expensive, the CFD solution $c_{p}^{\mathrm{CFD}}$ is replaced by its surrogate model approximation $\hat{c}_{p}^{\mathrm{CFD}}$ to give the objective function

$$
\operatorname{RMSE}_{\mathcal{J}}(x):=\sqrt{\frac{1}{|\mathcal{J}|} \sum_{i \in \mathcal{J}}\left(\hat{e}_{i}(x)\right)^{2}}=\sqrt{\frac{1}{|\mathcal{J}|} \sum_{i \in \mathcal{J}}\left|\left(c_{p}^{\mathrm{WTT}}\right)_{i}-\left(\hat{c}_{p}^{\mathrm{CFD}}(x)\right)_{\mathcal{I}(i)}\right|^{2}} .
$$

The optimal CFD input parameter $x^{*}=\left(\mathrm{M}_{*}, \alpha_{*}\right)$ with respect to this quantity is then defined as its global minimum in the parameter space $\mathcal{D}$,

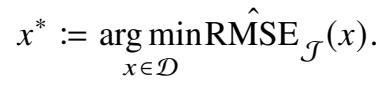

The corrected $c_{p}$-distribution can be assessed by evaluating the surrogate model at this parameter combination,

$$
c_{p}^{\mathrm{corr}}:=\hat{c}_{p}^{\mathrm{CFD}}\left(x^{*}\right)
$$

Note that the surrogate model provides the full surface $c_{p}$-distribution by minimizing the differences to the experimental data at the six sections. Hence, an integration of $c_{p}$ to obtain global coefficients is possible. Following this approach, we can account for integral forces and moments by simply defining mathematical constraints which supplement the objective function in Eq. (10) resulting in a constraint optimization problem. To compute integral coefficients for arbitrary input parameters $(\mathrm{M}, \alpha)$ during the optimization process, a second surrogate model for the skin friction coefficient $c_{f}$ is constructed. The surrogate models for drag and friction are then evaluated and the corresponding responses are integrated numerically over the surface of the aircraft to obtain the corresponding integral aerodynamic coefficients. Note that the stake which intersects the aircraft geometry introduces an error in the surface integration. A correction can be obtained by extrapolating the surface distributions to the shadow of the stake. According to NLR, this approach yields corrections for the lift and drag coefficient in the order of $10^{-4}$ and $10^{-5}$, respectively, which are therefore neglected throughout this case study.

\section{Results}

The results of the described correction approach are presented in this chapter, using the uncorrected free stream conditions and aerodynamic coefficients from the wind tunnel. Following the notation in Table 1, we will - in the remainder of this work - refer to the set of pressure taps at section cut $i$ as $\mathcal{J}_{i}, i=1, \ldots, 6$, while $\mathcal{J}_{\text {all }}$ denotes the set of all pressure taps, $\mathcal{J}_{\text {all }}=\cup_{i} \mathcal{J}_{i}$. Three different studies are carried out:

1) Optimization over all pressure taps: The above approach was applied to the experimental data taking all pressure taps into account, i.e. setting $\mathcal{J}=\mathcal{J}_{\text {all }}$.

2) Optimization over individual section cuts: The objective function was defined on the pressure taps of the individual section cuts only, i.e. setting $\mathcal{J}=\mathcal{J}_{i}, i=1, \ldots, 6$.

3) Optimization subject to constraints: The optimization task was again defined over all pressure taps choosing $\mathcal{J}=\mathcal{J}_{\text {all }}$ but additional constraints of matching the wind tunnel measured overall lift and/or drag coefficient were introduced.

\section{A. Optimization over all pressure taps}

The above approach was applied to the wind tunnel measurements choosing $\mathcal{J}=\mathcal{J}_{\text {all }}$, i.e. taking all pressure taps into account. The absolute corrections, the corresponding objective function values $\operatorname{RMSE}_{\mathcal{J}_{\text {all }}}\left(x^{*}\right)$ as well as the 
Table 3 Results of the correction approach when optimizing over all pressure taps.

\begin{tabular}{|c|c|c|c|c|c|}
\hline \multicolumn{2}{|c|}{ uncorrected } & \multicolumn{2}{|c|}{ corrections } & \multirow{2}{*}{$\operatorname{RMSE}_{\mathcal{J}_{\text {all }}}\left(x^{*}\right)$} & \multirow{2}{*}{$\begin{array}{l}\text { improvem. in } \\
\operatorname{RMSE}_{\mathcal{J}_{\text {all }}}\end{array}$} \\
\hline $\mathrm{M}$ & $\alpha\left[^{\circ}\right]$ & $\Delta \mathrm{M}$ & $\Delta \alpha\left[^{\circ}\right]$ & & \\
\hline 0.8502 & 2.447 & -0.0005 & -0.068 & $5.28 \cdot 10^{-2}$ & $9.77 \%$ \\
\hline
\end{tabular}

improvement in the objective function values compared to the uncorrected input parameters, i.e. the wind tunnel measured parameters, are given in Table 3 .

Figure 6 shows the corrected $c_{p}$-distributions at the six wing sections, where pressure taps are located, in comparison to the $c_{p}$-distribution to the uncorrected parameters and the measurement data for the wind tunnel test.

Whereas the predicted CFD solutions shows good agreement with the measured wind tunnel data at the four inner cuts (upper four plots), larger discrepancies are observed for the outer two cuts (lower two plots). However, compared to the uncorrected CFD solution, the resulting corrected $c_{p}$-distribution shows improved consistency with the wind tunnel measurements at all section cuts-especially in predicting the position and strength of the shock. 


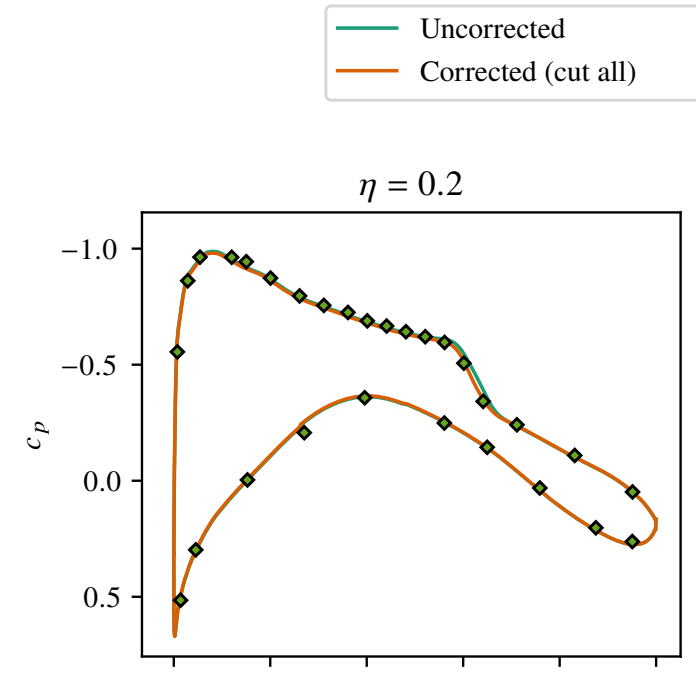

$\diamond \quad$ Experiment
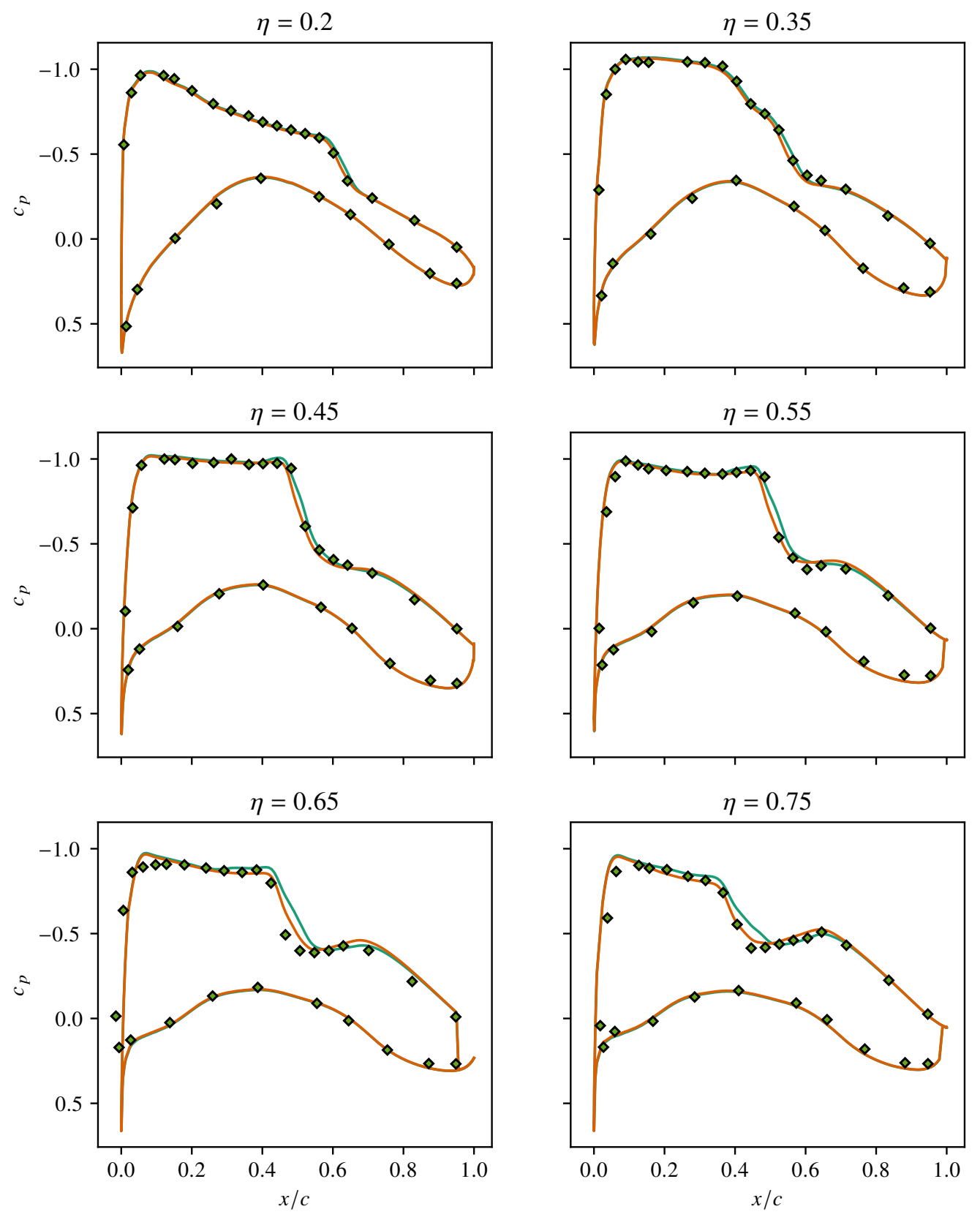

Figure 6 Corrected and uncorrected $c_{p}$-distributions for study 1 at the 6 section cuts. 


\section{B. Optimization for individual section cuts}

To investigate whether the match of CFD solution and wind tunnel data can be improved for the separate section cuts, six further studies were set up. To this end, the objective function was only defined on those pressure taps, which are located at an individual section cut, i.e. the objective function

$$
\operatorname{RMSE}_{\mathcal{J}_{i}}:=\sqrt{\frac{1}{\left|\mathcal{J}_{i}\right|} \sum_{i \in \mathcal{J}_{i}}\left|\left(c_{p}^{\mathrm{WTT}}\right)_{i}-\left(\hat{c}_{p}^{\mathrm{CFD}}(x)\right)_{I(i)}\right|^{2}}
$$

was considered for section cut $i=1, \ldots, 6$ (cf. Table 1 ).

The corresponding corrections for $\mathrm{M}=0.8502$ and $\alpha=2.4473^{\circ}$ were obtained by optimizing $\mathrm{RMSE}_{\mathcal{J}}$ for the respective pressure tap set $\mathcal{J}$. The corrections, the corresponding objective function value $\mathrm{RMSE}_{\mathcal{J}_{k}}\left(x^{*}\right)$ and, for comparison with each other, the overall root mean squared error RMSE $\hat{\mathcal{J}}_{\text {all }}\left(x^{*}\right)$ are given in Table 4

Table 4 Resulting corrections for study cases 2.1-2.6 in comparison to study 1 .

\begin{tabular}{|c|c|c|c|c|c|}
\hline \multirow{2}{*}{$\begin{array}{c}\text { section cut } \\
k\end{array}$} & \multirow{2}{*}{ study } & \multicolumn{2}{|c|}{ corrections } & \multirow{2}{*}{$\operatorname{RMSE}_{\mathcal{J}_{k}}\left(x^{*}\right)$} & \multirow{2}{*}{$\hat{\operatorname{RMSE}}_{\mathcal{J}_{\text {all }}}\left(x^{*}\right)$} \\
\hline & & $\Delta \mathrm{M}$ & $\Delta \alpha\left[^{\circ}\right]$ & & \\
\hline all & 1 & -0.0005 & -0.0680 & $5.28 \cdot 10^{-2}$ & $5.28 \cdot 10^{-2}$ \\
\hline 1 & 2.1 & -0.0013 & +0.0002 & $1.30 \cdot 10^{-2}$ & $5.46 \cdot 10^{-2}$ \\
\hline 2 & 2.2 & +0.0002 & -0.0579 & $2.52 \cdot 10^{-2}$ & $5.42 \cdot 10^{-2}$ \\
\hline 3 & 2.3 & +0.0021 & -0.1698 & $2.86 \cdot 10^{-2}$ & $5.81 \cdot 10^{-2}$ \\
\hline 4 & 2.4 & +0.0056 & -0.3011 & $4.94 \cdot 10^{-2}$ & $5.82 \cdot 10^{-2}$ \\
\hline 5 & 2.5 & -0.0032 & -0.0166 & $8.74 \cdot 10^{-2}$ & $5.88 \cdot 10^{-2}$ \\
\hline 6 & 2.6 & -0.0002 & -0.0860 & $5.54 \cdot 10^{-2}$ & $5.30 \cdot 10^{-2}$ \\
\hline
\end{tabular}

In order to provide a quantitative comparison of the results, the predicted root mean squared errors at the individual section cuts as well as the overall root mean squared error for all studies are given in Table 5.

Table 5 Results of the correction approach for study 2.1-2.6 in comparison to study 1 . The objective function value of the respective optimization process is highlighted in gray.

\begin{tabular}{|c|c|c|c|c|c|c|c|}
\hline \multirow{2}{*}{ exp. } & \multicolumn{7}{|c|}{$\hat{\operatorname{RME}}_{\mathcal{J}}\left(x^{*}\right) \cdot 10^{2}$} \\
\cline { 2 - 8 } & $\mathcal{J}=\mathcal{J}_{1}$ & $\mathcal{J}=\mathcal{J}_{2}$ & $\mathcal{J}=\mathcal{J}_{3}$ & $\mathcal{J}=\mathcal{J}_{4}$ & $\mathcal{J}=\mathcal{J}_{5}$ & $\mathcal{J}=\mathcal{J}_{6}$ & $\mathcal{J}=\mathcal{J}_{\text {all }}$ \\
\hline 1 & 1.42 & 2.67 & 3.57 & 5.32 & 9.19 & 5.56 & 5.28 \\
2.1 & 1.30 & 2.74 & 3.65 & 5.60 & 9.32 & 6.04 & 5.46 \\
2.2 & 1.50 & 2.52 & 3.18 & 5.31 & 9.69 & 5.76 & 5.42 \\
2.3 & 2.37 & 3.08 & 2.86 & 5.24 & 10.60 & 6.04 & 5.81 \\
2.4 & 3.49 & 4.10 & 3.23 & 4.94 & 10.15 & 5.85 & 5.82 \\
2.5 & 1.86 & 3.77 & 5.26 & 6.53 & 8.74 & 6.41 & 5.88 \\
2.6 & 1.49 & 2.67 & 3.55 & 5.29 & 9.26 & 5.54 & 5.30 \\
\hline
\end{tabular}

As one can see from Table Table 5, improvements over the results of study 1 can be obtained via matching for the individual section cuts. However, these improvements are always at the expense of the matching for the other cuts. The established optimum $\Delta \mathrm{M}$ and $\Delta \alpha$ values per section do not show a smooth trend in spanwise direction, with the angle of attack correction in particular showing large spread amongst the six sections. 


\section{Optimization with subject to constraints}

Besides a good match with the measured wind tunnel $c_{p}$-distribution, one may wish to obtain a good consistency with the measured overall forces and moments. For the third study, we therefore impose additional constraints of matching integral coefficients on the optimization problem. For this purpose, an additional surrogate model was built for the viscous shear stress following the approach outlined in Section IV.A. Hence also viscous contributions are consistently taken into account even though only distributed pressures are available from the experiment. To compute the integral coefficients for a given CFD surrogate model approximation, the responses are integrated numerically over the surface of the discretized aircraft model.

Figure 7]shows the CFD lift, drag and pitching moment coefficient approximation with respect to the Mach number M and the angle of attack $\alpha$.
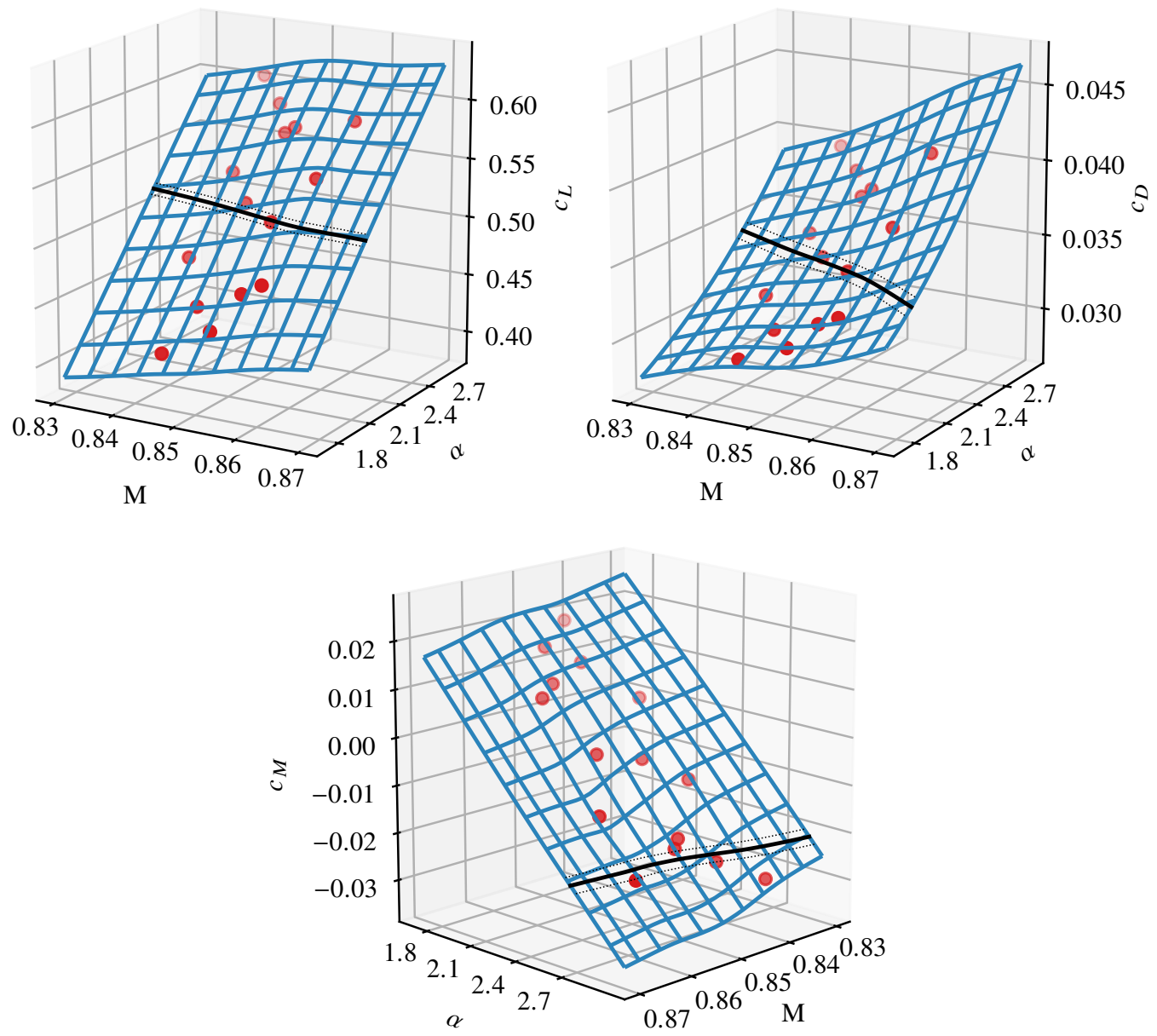

Figure 7 Lift coefficient (upper left), drag coefficient (upper right) and pitching moment coefficient (bottom) based on the CFD surrogate models with respect to Mach number and angle of attack.

The red dots indicate the corresponding values for the CFD snapshots, while the black lines are defined by the $\alpha$-M-combinations for which the CFD approximation equals the integral coefficients measured in the wind tunnel of $c_{L}^{\mathrm{WTT}}=0.5154, c_{D}^{\mathrm{WTT}}=0.0342$ and $c_{M}^{\mathrm{WTT}}=-0.0216$. They are complemented by the black dotted curves which indicate the uncertainty bounds due to the accuracy of the measuring instruments, cf. Table 2

The projections of the curves in the M- $\alpha$-plane are shown in Figure 8 As one can see in the figure, there is no $\mathrm{M}-\alpha$-combination for which the approximation of all three integral coefficients exactly match the wind tunnel measured values. While there is a domain in the $\alpha$-M-plane where the approximate CFD solution matches the wind tunnel measured lift and drag coefficient within their uncertainty ranges, it is not possible to match the wind tunnel measured 


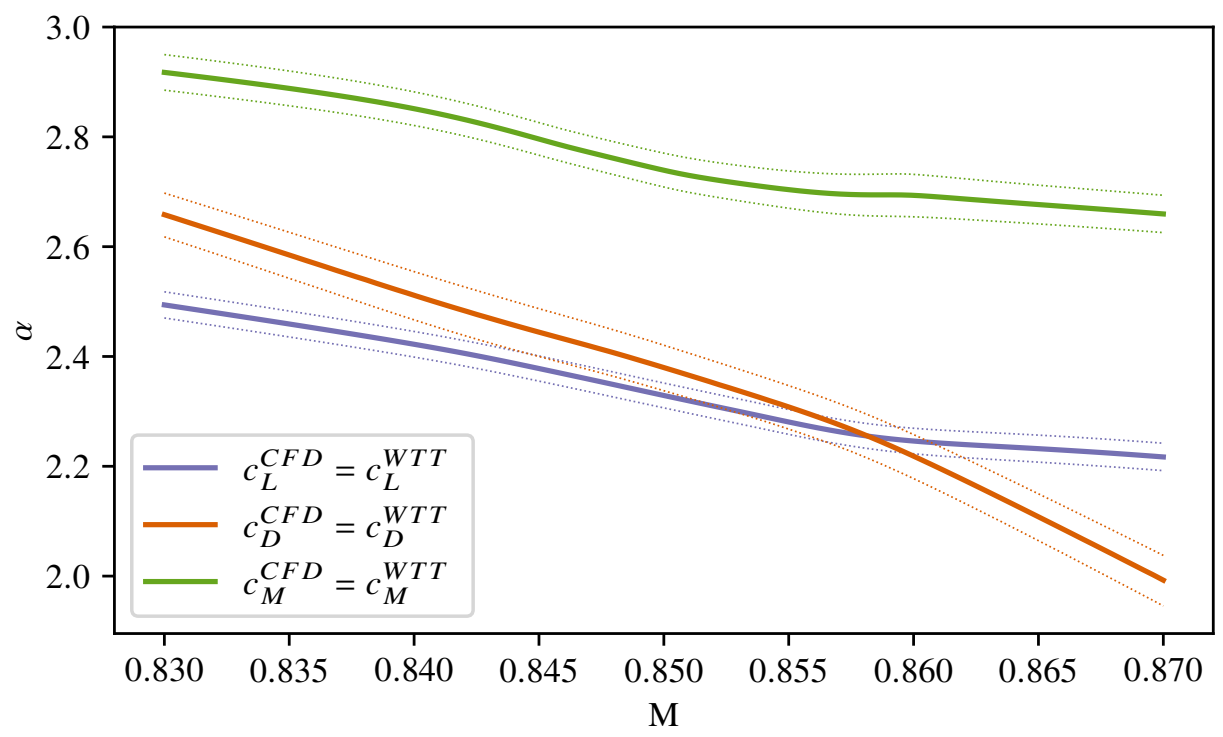

Figure 8 Plot of the $\alpha$-M-combinations for which the approximation of the CFD lift, drag and pitching moment coefficient match the corresponding wind tunnel results. The thin dotted curves indicate the uncertainty bounds in the integral coefficients due to instrumentation in the wind tunnel.

pitching moment coefficient at the same time. This serves as an indication that the wall interference effects are not constant along the model's $x$-axis. The presence of the walls is likely to cause the horizontal tail to experience a different local angle of attack correction than the wing causing pitching moment differences.

Following this observation, three studies were set up:

3.1. The optimization problem of study 1 is restricted to $\mathrm{M}-\alpha$-combinations for which the approximation to the CFD lift coefficient equals - except for instrumental uncertainty - the wind tunnel result, i.e. $\left|c_{L}^{\mathrm{CFD}}-c_{L}^{\mathrm{WTT}}\right| \leq 0.0038$.

3.2. The optimization problem of study 1 is restricted to $\mathrm{M}-\alpha$-combinations for which the approximation to the CFD drag coefficient equals - except for instrumental uncertainty - the the wind tunnel result, i.e. $\left|c_{D}^{\mathrm{CFD}}-c_{D}^{\mathrm{WTT}}\right| \leq 0.0004$.

3.3. Mach number and angle of attack are determined such that the CFD approximation yields - except for instrumental uncertainty - the same lift and drag coefficient as measured in the wind tunnel test, i.e. $\left|c_{L}^{\mathrm{CFD}}-c_{L}^{\mathrm{WTT}}\right| \leq 0.0038$ and $\left|c_{D}^{\mathrm{CFD}}-c_{D}^{\mathrm{WTT}}\right| \leq 0.0004$.

The correction results were obtained via a constraint numerical optimization. In all cases the numerical optimizer terminated successfully and a solution, which fulfills the imposed constraints was found. Table 6 summarizes the results of study 3.1-3.3 in comparison to the result of study 1 .

Table 6 Results of study 3 in comparison to study 1.

\begin{tabular}{|c|c|c|c|c|c|}
\hline \multirow{2}{*}{ exp. } & \multicolumn{2}{|c|}{ corrections } & \multirow{2}{*}{$c_{L}$} & \multirow{2}{*}{$c_{D}$} & $\mathrm{RMSE}_{\mathcal{J}_{\text {all }}}\left(x^{*}\right)$ \\
\cline { 2 - 4 } & $\Delta \mathrm{M}$ & $\Delta \alpha\left[^{\circ}\right]$ & & & $5.28 \cdot 10^{-2}$ \\
\hline 1 & -0.0005 & -0.0680 & 0.5226 & 0.0348 & $5.28 \cdot 10^{-2}$ \\
3.1 & -0.0002 & -0.0962 & 0.5192 & 0.0339 & $5.31 \cdot 10^{-2}$ \\
3.3 & -0.0005 & -0.0679 & 0.5233 & 0.0342 & $5.28 \cdot 10^{-2}$ \\
\hline
\end{tabular}

The black star in Figure 9 visualizes the obtained correction values in study 3.3 in a close up of the M- $\alpha$-plane in Figure 8. The green shaded area indicates the domain where the approximate CFD solution matches the wind tunnel measured lift and drag coefficient except for the instrumental uncertainties.

Note that the corrections determined in study 3.2 differ from those of study 1 by only $0.0001^{\circ}$ in the angle of attack. 


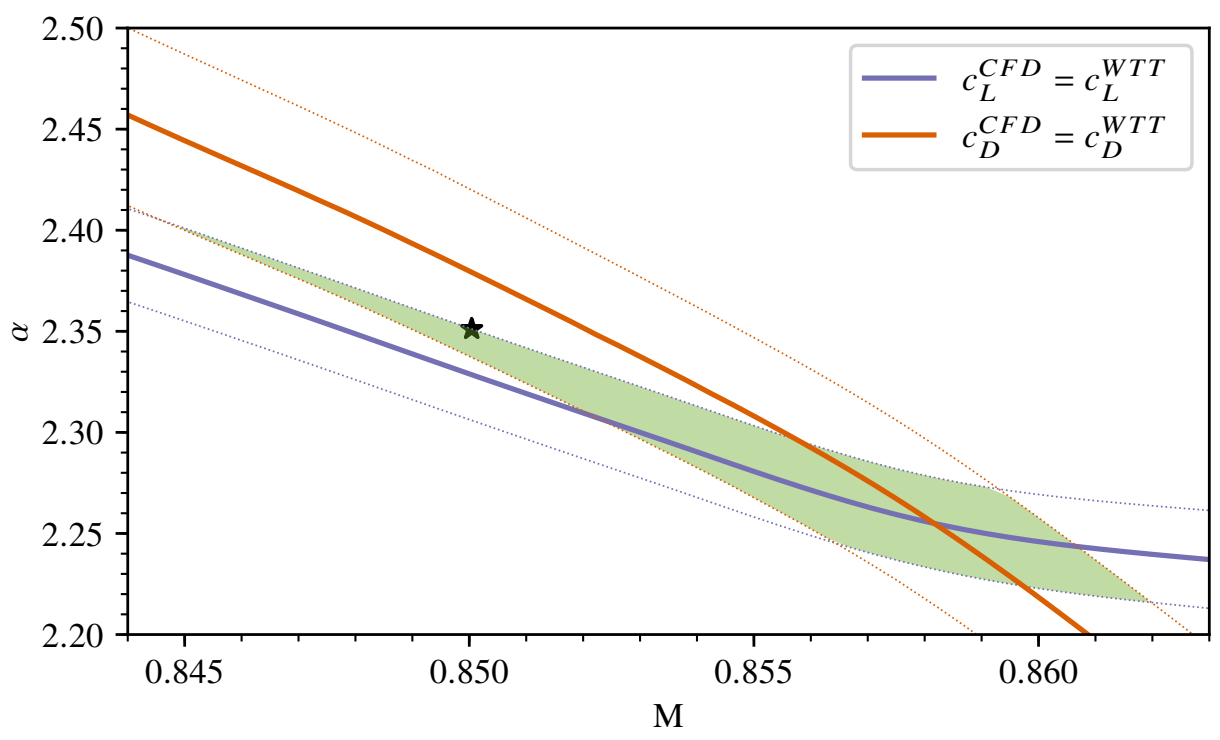

Figure 9 Close up of Figure 8. The black star marks the optimal solution for the $c_{L}$ and $c_{D}$ constrained optimization problem of study 3.3. The green shaded intersection area indicates the domain where the approximate solutions match the wind tunnel measured lift and drag coefficient within the associated uncertainty bounds.

Furthermore, study 3.3 results in the same corrections as study 3.1 as the approximated drag coefficient of the optimal soultion of study 3.1 is within the uncertainty bounds of the wind tunnel measured drag coefficient. Figure 10 shows the corrected $c_{p}$-distributions at the six section cuts for study 3.1/3.3. For comparison, the resulting $c_{p}$-distribution of study 1 is also plotted. As one can see from the plots, imposing the constraints has close to no effect on the pressure distributions at the 6 section cuts-only the pressure level in front of the shock location is slightly decreased in order to match the integral coefficients. This impression is confirmed by the fact that the overall root mean squared error increases by only $0.5 \%$ compared to the unconstrained corrections found in study 1 . This also indicates that the found corrections in study 3.3 show a very good consistency with the wind tunnel measured data in terms of $c_{p}$. 


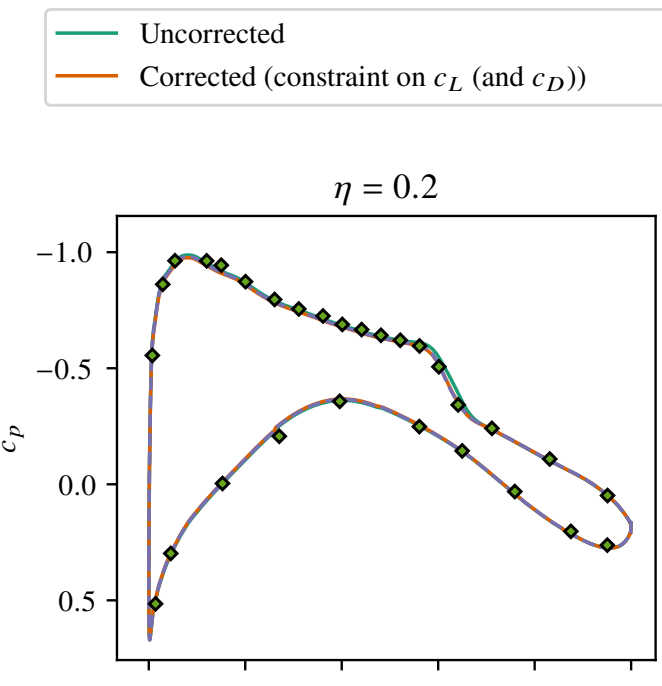

---. Corrected (no constraint)

$\diamond \quad$ Experiment
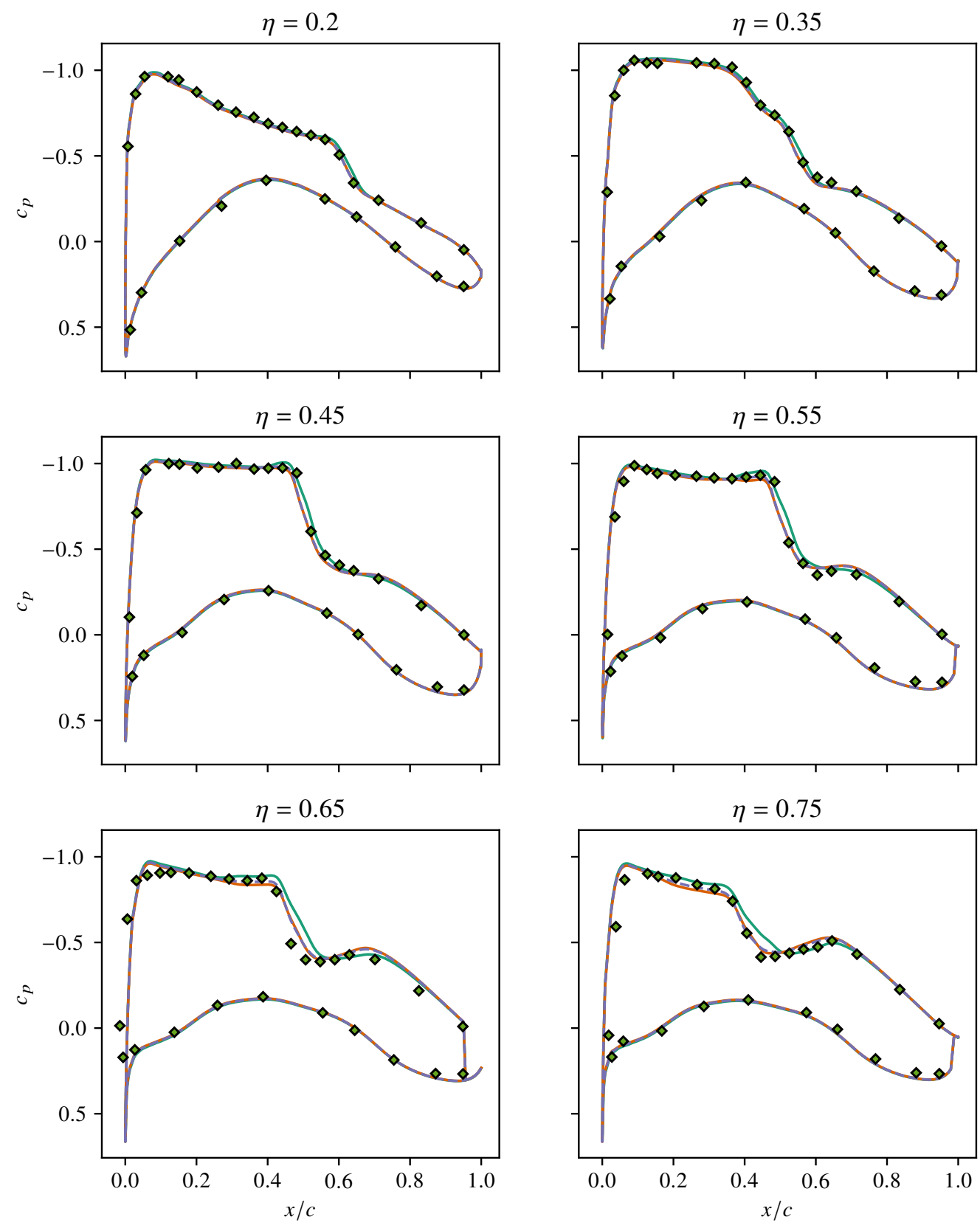

Figure 10 Corrected and uncorrected $c_{p}$-distributions for study 3.1./3.3. 


\section{Comparison against WIN3VE}

As a sanity check the free stream corrections obtained in the previous sections are compared with the predictions from the WIN3VE pressure signature method (see Section III.A). For the specific flow condition studied, WIN3VE yields estimates of $-0.04^{\circ}$ for the the wall-induced bias error on angle of attack and -0.001 for the Mach number. Figure 11 shows both the uncorrected and WIN3VE corrected wind tunnel data, as well as same wind tunnel data corrected with the results from studies 1 (optimization over all wing pressures) and 3.3 (optimization subjected to both $c_{L}$ and $c_{D}$ constraints). Study 1 produces a result that is closest to the pressure signature method prediction, whereas study 3.3 remains further off. This might be due to wall interference effects not being constant along the model (e.g. towards the tails): something that is more noticeable in the overall load coefficients, but less so in the wing pressures. Overall, both the pressure signature method and the minimization studies confirm that the $\Delta \mathrm{M}$ correction is practically zero (i.e. $<0.001$ ) and that the largest difference is in the angle of attack.
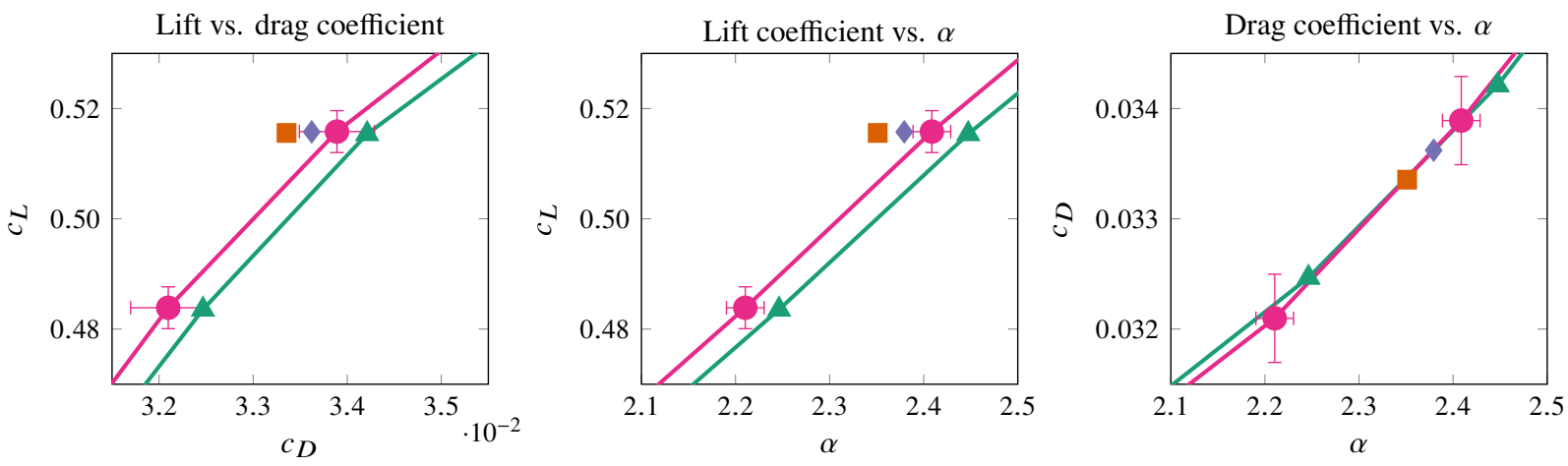

$\Delta$ Uncorrected $\diamond \quad$ Corrected (Study 1) $\quad$ Corrected (Study 3.3) $\quad$ Corrected (WIN3VE)

Figure 11 Lift and drag coefficient results before and after correction application. Error bars represent the $\pm 2 \sigma$ uncertainty due to the instrumentation.

\section{Discussion and conclusion}

In this work, an alternative method for correction of wind tunnel data using free-flow RANS CFD simulations was introduced and applied to the case of a business jet aircraft model at cruise condition. The approach seeks for the combination of angle of attack and Mach number for which the CFD-based pressure coefficient distribution best matches the wind tunnel sensor data at 6 given wing section cuts. For the use within the optimization process, the computationally demanding CFD simulations were replaced by a reduced order model for the pressure coefficient distribution based on 15 CFD simulations.

Three different studies have been carried out. For the first study, the root mean square error minimization was performed using the sensor data from all available 6 section cuts. The results show an improved agreement with the sensor data at all 6 section cuts. The reduced order model results were found to be able to accurately predict the strength and position of the shock wave for this test case. While a very good consistency with the sensor data was observed for the inner four section cuts, larger discrepancies occur at the outer two cuts. The $\Delta \alpha$ and $\Delta \mathrm{M}$ obtained in the study 1 are $-0.068^{\circ}$ and -0.0005 , implying that the largest discrepancy is in angle of attack.

The second study comprised of optimizations using the different section cuts individually. It was shown that in comparison to the result of study 1, the root mean squared error per section can be further reduced. However, each section improvement was at the expense of the other cuts. The established optimum $\Delta \alpha$ and $\Delta \mathrm{M}$ values per section did not reveal a smooth trend in spanwise direction. This could be a hint that perhaps the wing shape in the CFD simulation does not sufficiently resemble the actual wind tunnel model under loaded conditions. Besides a minimization based on wing pressure distributions only, one may also aim to include the overall forces and moments data. For this purpose, additional constraints for matching the integral coefficients where imposed on the optimization problem in a third study. Via this approach, corrections for which the CFD predictions match the wind tunnel lift and drag coefficients could be obtained. In this case, the overall root mean squared error of the wing pressures increased by only $0.5 \%$ in comparison to the unconstrained optimization from the first study, indicating that a good agreement with the measured pressure 
coefficient data could be obtained. With the additional lift and drag coefficient constraints in place, the corrections on Mach number and angle of attack are -0.0002 and $-0.096^{\circ}$.

A final comparison of the obtained free stream corrections against those obtained via a wall pressure signature method confirms that both the $\Delta \mathrm{M}$ and $\Delta \alpha$ correction are to be of negative sign. Both the pressure signature method and our minimization studies confirm that the $\Delta \mathrm{M}$ correction is practically zero $(<0.001)$, which suggests that the procedure for setting the Mach number was adequate. The largest uncertainty is in the angle of attack, with predicted correction values ranging from $-0.04^{\circ}$ with the wall pressure signature method down to $-0.068^{\circ}$ according to our optimization over all wing pressures simultaneously.

In this case study, the applicability of the proposed data correction approach was successfully demonstrated for a business jet at transonic cruise condition. Further research needs to be done to investigate the performance of the approach for a larger range of flight conditions. While in this study, a fixed deformed model geometry was used to generate the CFD data, the investigation of different flight conditions may require to take different aeroelastic deformations into account. For more definite wall interference conclusions, a next step could be to repeat the demonstrated approach, but instead of using measured sensor data rely on in-tunnel CFD simulations. This would mitigate some of the geometric and potentially turbulence model uncertainties inherent to the current case study setup.

\section{Acknowledgment}

The authors kindly thank the organizers of the CAE-DNW workshop for the opportunity to use the AVM experimental data along with the standardized CFD grid for this study.

\section{References}

[1] Ewald, B. F. R., Wind tunnel wall correction: La Correction des effets de paroi en soufflerie, AGARDograph, Vol. 336, AGARD, North Atlantic Treaty Organization and [Available from] NASA Center for Aerospace Information, Neuilly-sur-Seine Cedex, France and Hanover, MD, 1998.

[2] Walker, N., A.S. Wiseman, "The Pressure Signature Method for Blockage Corrections, and it Application to the Industrial Wind Tunnel,” NLR Technical report TR 88072 U Report No. BU-263, University of Bristol, Dept. of Aeronautical Engineering, 1981.

[3] Ulbrich, N., and Boone, A., "Direct Validation of the Wall Interference Correction System of the Ames 11-Foot Transonic Wind Tunnel: NASA/TM-2003-212268,", 2003.

[4] Wubben, F. J. M., and Maseland, H., "Verification of wind tunnel model support and wall interference assessments in DNW-HST by CFD simulations," NATO STO-MP-AVT-284: Advanced Wind Tunnel Boundary Simulation, edited by NATO, 2018.

[5] Neyland, V., Bosniakov, S., Glazkov, S., Ivanov, A., Matyah, S., Mikhailov, S., and Vlasenko, V., "Conception of electronic wind tunnel and first results of its implementation," Progress in Aerospace Sciences, Vol. 37, No. 2, 2001, pp. 121-145. doi:10.1016/S0376-0421(00)00013-0.

[6] Melber-Wilkending, S., Heidebrecht, A., and Wichmann, G., "CFD Validation by Wind Tunnel Measurements: Uncertainty Assessment by Numerical Simulation of Complete Wind Tunnel Flows," NATO MP-AVT-147-22: Computational Uncertainty in Military Vehicle Design, edited by NATO, 2007.

[7] König, B., Fares, E., and Wright, M. C., "Lattice Boltzmann Simulation of the ETW Slotted Wall Test Section," NATO STO-MP-AVT-284: Advanced Wind Tunnel Boundary Simulation, edited by NATO, 2018.

[8] Rivers, S. M., Nayani, S. N., Tinetti, A. F., Brynildsen, S. E., and Ferris, R. J., "Numerical Study of the High-Speed Leg of the National Transonic Facility," NATO STO-MP-AVT-284: Advanced Wind Tunnel Boundary Simulation, edited by NATO, 2018.

[9] Morrison, J. H., Kleb, W. L., and Vassberg, J. C., "Observations on CFD Verification and Validation from the AIAA Drag Prediction Workshops," 52nd Aerospace Sciences Meeting, American Institute of Aeronautics and Astronautics, Reston, Virginia, 2014, p. B4. doi:10.2514/6.2014-0202.

[10] Vassberg, J. C., "Challenges and Accomplishments of the AIAA CFD Drag Prediction Workshop Series," NATO STO-MP-AVT246: Progress and Challenges in Validation Testing for Computational Fluid Dynamics, edited by NATO, 2016. 
[11] Pinnau, R., "Model Reduction via Proper Orthogonal Decomposition," Model Order Reduction: Theory, Research Aspects and Applications, Mathematics in Industry, Vol. 13, edited by H.-G. Bock, F. de Hoog, A. Friedman, A. Gupta, H. Neunzert, W. R. Pulleyblank, T. Rusten, F. Santosa, A.-K. Tornberg, L. L. Bonilla, R. Mattheij, O. Scherzer, W. H. A. Schilders, H. A. van der Vorst, and J. Rommes, Springer Berlin Heidelberg, Berlin, Heidelberg, 2008, pp. 95-109. doi:10.1007/978-3-540-78841-6_5.

[12] Holmes, P., Lumley, J. L., Berkooz, G., and Rowley, C. W., Turbulence, coherent structures, dynamical systems and symmetry, $2^{\text {nd }}$ ed., Cambridge monographs on mechanics, Cambridge University Press, Cambridge, 2012.

[13] Everson, R., and Sirovich, L., "Karhunen-Loève procedure for gappy data," Journal of the Optical Society of America A, Vol. 12, No. 8, 1995, p. 1657. doi:10.1364/JOSAA.12.001657.

[14] Bui-Thanh, T., Damodaran, M., and Willcox, K. E., "Aerodynamic Data Reconstruction and Inverse Design Using Proper Orthogonal Decomposition,” AIAA Journal, Vol. 42, No. 8, 2004, pp. 1505-1516. doi:10.2514/1.2159.

[15] Franz, T., and Held, M., "Data Fusion of CFD Solutions and Experimental Aerodynamic Data," ODAS 2017, edited by DLR and ONERA, 2017.

[16] Mifsud, M., Vendl, A., Hansen, L.-U., and Görtz, S., "Fusing wind-tunnel measurements and CFD data using constrained gappy proper orthogonal decomposition," Aerospace Science and Technology, Vol. 86, 2019, pp. 312-326. doi:10.1016/j.ast.2018.12. 036.

[17] Bertram, A., Bekemeyer, P., and Held, M., "Fusing distributed aerodynamic data using Bayesian Gappy Proper Orthogonal Decomposition," To appear in AIAA Aviation 2021 Forum, American Institute of Aeronautics and Astronautics, 2021.

[18] Jouhaud, J.-C., Sagaut, P., and Labeyrie, B., “A Kriging Approach for CFD/Wind-Tunnel Data Comparison,” Computers \& Geosciences, Vol. 128, No. 4, 2006, pp. 847-855. doi:10.1115/1.2201642.

[19] Ma, B., Wang, G., Ye, Z., and Xu, L., "A numerical Method for Transonic Wind Tunnel Wall Interference Correction in Airfoil Testing," 34th AIAA Applied Aerodynamics Conference, American Institute of Aeronautics and Astronautics, Reston, Virginia, 2016. doi:10.2514/6.2016-3575.

[20] Belligoli, Z., Dwight, R., and Eitelberg, G., “Assessment of a Data Assimilation Technique for Wind Tunnel Wall Interference Corrections," AIAA Scitech 2019 Forum, American Institute of Aeronautics and Astronautics, Reston, Virginia, 2019. doi:10.2514/6.2019-0939.

[21] Belligoli, Z., Dwight, R., and Eitelberg, G., "RANS Data Assimilation Techniques for Wind-Tunnel Wall Interference Corrections," AIAA Aviation 2019 Forum, American Institute of Aeronautics and Astronautics, Reston, Virginia, 2019. doi:10.2514/6.2019-2976.

[22] Braconnier, T., Ferrier, M., Jouhaud, J.-C., Montagnac, M., and Sagaut, P., “Towards an adaptive POD/SVD surrogate model for aeronautic design," Computers \& Fluids, Vol. 40, No. 1, 2011, pp. 195-209. doi:10.1016/j.compfluid.2010.09.002.

[23] Ly, H. V., and Tran, H. T., "Modeling and control of physical processes using proper orthogonal decomposition," Mathematical and Computer Modelling, Vol. 33, No. 1-3, 2001, pp. 223-236. doi:10.1016/S0895-7177(00)00240-5.

[24] Walton, S., Hassan, O., and Morgan, K., "Reduced order modelling for unsteady fluid flow using proper orthogonal decomposition and radial basis functions," Applied Mathematical Modelling, Vol. 37, No. 20-21, 2013, pp. 8930-8945. doi:10.1016/j.apm.2013.04.025.

[25] Zimmermann, R., “A Locally Parametrized Reduced-Order Model for the Linear Frequency Domain Approach to TimeAccurate Computational Fluid Dynamics," SIAM Journal on Scientific Computing, Vol. 36, No. 3, 2014 , pp. B508-B537. doi:10.1137/130942462.

[26] Franz, T., Zimmermann, R., Görtz, S., and Karcher, N., "Interpolation-based reduced-order modelling for steady transonic flows via manifold learning," International Journal of Computational Fluid Dynamics, Vol. 28, No. 3-4, 2014 , pp. 106-121. doi:10.1080/10618562.2014.918695.

[27] Hua, J., Zheng, S., Zhong, M., Wang, G., Chu, L., Liu, F., and Bai, J., "Design and Verification Study of an Aerodynamic Validation Model," 7th Asia-Pacific International Symposium on Aerospace Technology, 2015, pp. 25-27.

[28] Hua, J., Zheng, S., Zhong, M., Wang, G., Eitelberg, G., Hegen, S., and Gebbink, R., "Recent development of a CFD-wind tunnel correlation study based on CAE-AVM investigation," Chinese Journal of Aeronautics, Vol. 31, No. 3, 2018 , pp. 419-428. doi:10.1016/j.cja.2018.01.017. 
[29] Zhong, M., Zheng, S., Wang, G., Hua, J., and Gebbink, R., "Correlation analysis of combined and separated effects of wing deformation and support system in the CAE-AVM study," Chinese Journal of Aeronautics, Vol. 31, No. 3, 2018 , pp. 429-438. doi:10.1016/j.cja.2018.01.015.

[30] Kursakov, I., Kazhan, E., and Gebbink, R., "Computational study of wing deformation and sting interference effects with the CAE-AVM test case," Chinese Journal of Aeronautics, Vol. 31, No. 10, 2018, pp. 1954-1961. doi:10.1016/j.cja.2018.05.018.

[31] Gebbink, R., Wang, G., and Zhong, M., "High-speed Wind Tunnel Test of the CAE-AVM in DNW-HST for CFD Validation Purposes," 55th AIAA Aerospace Sciences Meeting, American Institute of Aeronautics and Astronautics, Reston, Virginia, 2017, p. 2013. doi:10.2514/6.2017-0332.

[32] Elsenaar, B., Philipsen, I., and Poel, M., "DNW-HST (High Speed Tunnel) 50-Year Anniversary,” 48th AIAA Aerospace Sciences Meeting Including the New Horizons Forum and Aerospace Exposition, American Institute of Aeronautics and Astronautics, Reston, Virigina, 2010, p. 493. doi:10.2514/6.2010-575.

[33] Gebbink, R., Wang, G., and Zhong, M., "High-speed wind tunnel test of the CAE aerodynamic validation model," Chinese Journal of Aeronautics, Vol. 31, No. 3, 2018, pp. 439-447. doi:10.1016/j.cja.2018.01.010.

[34] Gebbink, R., Kapteijn, K., Bai, F., Mao, K., Zhang, D., Ba, Y., and Zhang, M., "Consistency Verifications of the DNW-HST Tunnel Interference Correction Bookkeeping," 2018 AIAA Aerospace Sciences Meeting, American Institute of Aeronautics and Astronautics, Reston, Virginia, 2018, p. 493. doi:10.2514/6.2018-2116.

[35] Labrujère, T. E., Maarsingh, R. A., and Smith, J., "Evaluation of Measured-Boundary-Condition methods for 3D subsonic wall interference," NLR Technical report TR 88072 U, NLR, 1988.

[36] German-Dutch Wind Tunnels, "CAE-DNW Workshop on CFD - Wind Tunnel Correlation Study,” , March 2016. URL http://cae-dnw-workshop.dnw.aero/cases

[37] Kok, J. C., Boerstoel, J. W., Kassies, A., and Spekreijse, S. P., “A Robust Multi-Block Navier-Stokes Flow Solver for Industrial Applications,” Tech. Rep. NLR-TP-1996-323, Netherlands Aerospace Centre (NLR), 1996.

[38] Kok, J. C., "A high-order low-dispersion symmetry-preserving finite-volume method for compressible flow on curvilinear grids," Journal of Computational Physics, Vol. 228, No. 18, 2009, pp. 6811 - 6832. doi:10.1016/j.jcp.2009.06.015.

[39] Kok, J. C., and Spekreijse, S. P., "Efficient and Accurate Implmentation of the $k-\omega$ Turbulence Model in the NLR Multi-Block Navier-Stokes System,” Tech. Rep. NLR-TP-2000-144, Netherlands Aerospace Centre (NLR), 2000.

[40] Dol, H. S., Kok, J. C., and Oskam, B., “Turbulence Modelling for Leading-Edge Vortex Flows,” AIAA 40th Aerospace Sciences Meeting \& Exhibit, 2002. doi:10.2514/6.2002-843.

[41] Buhmann, M. D., Radial Basis Functions, Cambridge University Press, Cambridge, 2003. doi:10.1017/CBO9780511543241. 